\title{
Obtaining High Quality SCAL Data: Combining Different Measurement Techniques, Saturation Monitoring, Numerical Interpretation and Continuous Monitoring of Experimental Data
}

\author{
Yingxue Wang1,*, Shehadeh K. Masalmeh² \\ ${ }^{1}$ Shell International E \& P, Inc. Houston, USA, ${ }^{2}$ Shell Abu Dhabi BV
}

\begin{abstract}
SCAL parameters (i.e., Relative Permeability and Capillary Pressure curves) are key inputs to understand and predict reservoir behavior in all phases of development. Techniques to measure relative permeability and capillary pressure have been well established and applied to a wide variety of core samples both from sandstone and carbonate reservoirs. On the other hand, we frequently encounter quality compromised data due to challenges in experimental procedures, lack of understanding of measurement techniques, and poor quality of raw data. As a result, relative permeability is often viewed as a parameter with large uncertainties and a fitting parameter in history matching. A special core analysis program was recently carried out on selected core samples from a deep-water sandstone reservoir in the Gulf of Mexico. In this frontier, relative permeability has been ranked among the top subsurface uncertainties. It greatly impacts the production forecast and field development plan. However, due to the high temperature, high salinity and fluid compatibility issues, the core measurements faced very specific challenges and a good relative permeability dataset has not been obtained in the past for this area. In this work, we demonstrate that a quality set of relative permeability data can be obtained through close collaboration across disciplines, a properly designed protocol, adequate engagement with the laboratory, timely $\mathrm{QA} / \mathrm{QC}$ of experimental raw data, and appropriate interpretation incorporating numerical simulations. Well-defined and constrained relative permeability curves have been derived with the combination of steady state and centrifuge techniques. The average trend can be described by a residual oil saturation of $22 \%$, end-point relative permeabilities of 0.6 and 0.2 to oil and water, respectively and Corey exponents between 2 and 3 .
\end{abstract}

\section{Introduction}

SCAL parameters (i.e., Relative Permeability and Capillary Pressure curves) are key inputs to understand and predict reservoir behavior in all phases of development. SCAL plays a key role in the selection, design, and optimization of oil recovery processes e.g. waterflood, enhanced oil recovery. High quality SCAL data acquisition and application requires proper experimental program design: the use of state-of-the-art SCAL capabilities and techniques, timely monitoring of the experiments in progress and $\mathrm{QC}$ of the raw data. SCAL projects are highly specialized and require the leadership of professionals with the necessary qualifications and expertise. Techniques to measure relative permeability and capillary pressure include core flooding and centrifuge. In a core flooding test, oil and water may be co-injected into a core plug sample at controlled fractional flow rates. With stable pressure and saturation, relative permeabilities to oil and water can be derived simultaneously; the so-called Steady State (SS) test. Another type of core flooding test, ie. the Unsteady State (USS) test, involves injecting water only at a constant rate and recording the oil production. The centrifuge technique is a method to measure capillary pressure (multi-speed centrifuge) or relative permeability (single speed centrifuge). In a centrifuge test, a core plug is saturated with one phase and spun around in a core holder filled with the other phase. The production of the expelled phase from the core plug by the centrifugal force is measured as a function of time. Different techniques have their own advantages and limitations. In an SS or USS test, relative permeabilities for two phases are measured simultaneously. Note that very low flow rates are needed to measure the tail-end of relative permeability, and such low rates are often constrained by the equipment capability. As a result, SS and USS generally measure relative permeability of $\sim 10-3$ or higher. However, the measured saturation range can be increased by using a bump flood rate at the end of the experiment in combination with numerical interpretation of the data. In addition, in an USS test, only the data after

* corresponding author: Yingxue.Wang@shell.com

(c) The Authors, published by EDP Sciences. This is an open access article distributed under the terms of the Creative Commons Attribution License 4.0 (http://creativecommons.org/licenses/by/4.0/). 
breakthrough of the invading phase can be used. Moreover, viscous fingering at high mobility ratios could further complicate the measurement. Capillary pressure plays a key role in fluid distribution, but is not measured in a core flooding SS or USS test. The capillary pressure must be measured separately to interpret a flooding test. The centrifuge technique can measure relative permeability using single speed or capillary pressure using multispeed. It can achieve higher capillary pressure than a displacement (or flooding) test, measure very low relative permeability, and provide more accurate information on residual saturation. Besides, centrifuge displacement is gravity stable with no viscous fingering. A main limitation of the centrifuge method is that only relative permeability of the expelled phase can be derived. The abovementioned techniques have been well established and applied to a wide variety of core samples both from sandstone and carbonate reservoirs. However, in practice, we frequently encounter less satisfactory relative permeability data. Several factors could have contributed to the compromised quality, including challenges in experimental procedures, nonrepresentative core sample selection, lack of understanding of measurement techniques, poor quality raw data, inadequate analysis or interpretation, etc. As a result, relative permeability is often viewed as a parameter with large uncertainties and a fitting parameter in history matching. In this paper, we describe a relative permeability program carried out recently on core samples from a deep-water sandstone reservoir in the Gulf of Mexico. We first outline the protocol and highlight the importance of proper design of the experiments. We then describe the program in steps with the focus on timely data review and QA/QC. For data analysis, we use numerical simulations to interpret individual tests and combine results from different methods. We also discuss the possible impact of sample heterogeneity and large viscous pressure on the steady state tests.

\section{Experimental program}

A SCAL program was recently carried out on selected core samples from a deep-water sandstone reservoir in the Gulf of Mexico. In this frontier, relative permeability has been ranked among the top subsurface uncertainties, as shown in the tornado chart in Figure 1. Both the initial oil production rate and the oil-water displacement efficiency are greatly driven by oil-water relative permeability due to the significant aquifer presence. From a field development point of view, evaluation of such important parameters as well productivity, and therefore well count and ultimate recovery requires a set of quality and representative oil-water relative permeability data. Quality relative permeability data has not been obtained in the past in this area for several reasons. In this new frontier, the reservoir temperature is over $300{ }^{\circ} \mathrm{F}$ and in situ formation brine is saturated with total dissolved solids (TDS) of about 410,000 mg/l. These subsurface conditions have imposed specific challenges to the core measurements. In previous SCAL measurements, we experienced tubing corrosion and equipment failure because of those challenges. Besides, core tests were also compromised by missing raw data, less than optimal design, and lack of timely result review. Figure 2 contains examples from both SS and centrifuge tests previously performed on core plugs from this area. In the SS test, pressure data in Figure 2 (a) showed that the injection during some oil-water rate ratios was too short to assess if steady state was indeed achieved. At each injection ratio, only one saturation profile was reported with large variations as depicted in Figure 2 (b). The core plug was at non-uniform initial saturation, which could be caused by local heterogeneities in the sample or improper initialization. At each ratio, displacement probably did not reach steady state, resulting in a large saturation gradient along the plug. In a single speed centrifuge test in Figure 2 (c), the speed of $600 \mathrm{rpm}$ (revolution per minute) was used. The corresponding capillary pressure $(\sim 1.2 \mathrm{psi})$ was too low to measure the true residual oil saturation. In a multispeed centrifuge test in Figure 2 (d), speed selection could have been improved to better resolve the production. Moreover, manually recorded production data could not provide the full production profile. In this program, we used both steady state and centrifuge techniques to measure relative permeability. A high-level protocol is listed below.

1) Fluid and core plug selection

2) Core cleaning

3) Basic property characterization

4) Core plug initialization

5) Wettability restoration, i.e. aging

6) Steady state and centrifuge tests

7) Data interpretation 


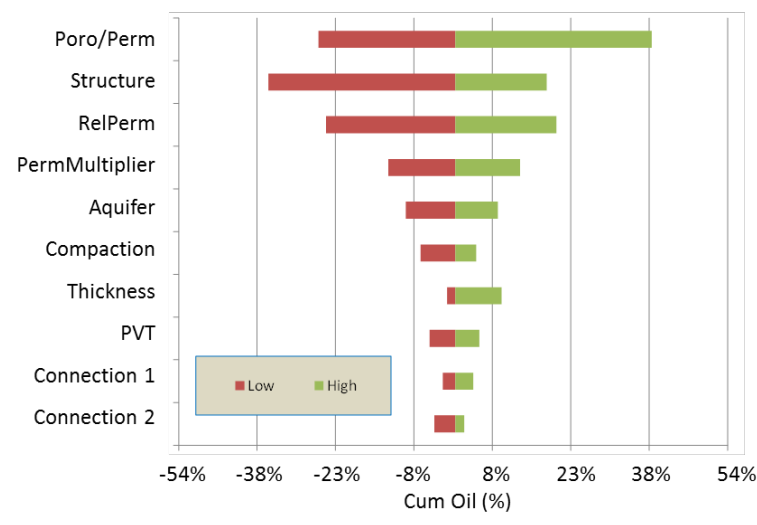

Figure 1: Impacts of subsurface uncertainties on cumulative oil production in the relevant reservoir in this study. Relative permeability is the third most sensitive parameters.

\subsection{Fluids and core plug selection, cleaning and characterization}

Dead crude oil from the same well and same depth interval was used in wettability restoration. As the reservoir live crude is relatively light with low GOR (< $500 \mathrm{scf} / \mathrm{bbl}$ ), dead crude is representative. Lab oil was used in initialization and in relative permeability tests to avoid any possible crude instability issues. The lab oil was selected based on its compatibility test results with the crude at different volumetric ratios. The in-situ TDS of the formation brine could not be achieved at experimental conditions with lower pressure. Instead, synthetic formation brine used in this program was diluted $40 \%$ with TDS of about $220,000 \mathrm{mg} / \mathrm{L}$. Two main rock fabrics were observed on cores in this reservoir. One with fine laminations has a wide range of permeability and is the main contributor to reservoir flow. The other fabric shows less organized features and is associated with lower permeability $(<20 \mathrm{mD})$. We selected core plugs primarily based on permeability and coverage of the two fabrics. The main flow capacity in the reservoir is contributed by the formation with permeability $10 \mathrm{mD}$ or higher. Core plugs lower than $10 \mathrm{mD}$ were not included in this program. Besides, the porosity-permeability trend from selected plugs reasonably followed those measured from routine core analysis (RCA). Then, plugs were paired based on permeability, porosity and rock fabrics so that one plug was tested using the SS method and one using the centrifuge method. The goal was to combine the results from each method to obtain a set of fully defined relative permeability curves, as recommended by Masalmeh and Jing [1]. To achieve this goal, a plug pair should have the same or very similar properties. Fourteen core plugs were selected in this program. Figure 3 shows CT images of two plugs representing two types of rock fabrics. Figure 4 compares the porosity and permeability of the selected plugs with those from RCA. Proper cleaning is necessary to remove any remaining hydrocarbon fluids and possible contamination of drilling fluid, and render core plugs water-wet. Core cleaning in this program was complicated by the existence of solid hydrocarbon $(\mathrm{HC})$ in the pore space. Our previous experience showed that aggressive solvents normally used in core analysis would keep stripping some solid HC off, but could not entirely remove it. It left large uncertainties in how much solid HC was left in pores. The cleaning protocol was therefore updated using the relatively benign solvent cyclohexane to preserve the solid HC. Thin section analysis on plug end-trims after cleaning confirmed the preservation of solid HC (Figure 5). Basic core properties were measured including dimensions, CT (computer-assisted tomography) scanning, porosity, permeability, XRD (X-ray diffraction), etc. CT scanning is essential to evaluate plug integrity and homogeneity. XRD provides minerology information. CT images and XRD data are available before start of the SCAL program. Plug dimensions, porosity, and stressed brine permeability are critical data in a relative permeability program. In a SCAL test, we prefer 1.5-inch diameter core plugs of $5 \mathrm{~cm}$ or longer. Larger size core samples help reduce the uncertainties in measuring small pore volume, and mitigate the impact from the potential capillary end effect during core flooding tests. In this program, due to limited availability of core samples, smaller core plugs samples were used with $\sim 1.5$-inch long and 1 inch in diameter. They are $10-$ $780 \mathrm{mD}$ in permeability and $17 \%-26 \%$ in porosity.

\subsection{Core plug initialization and wettability restoration}

Porous plate, centrifuge, and flooding are the most common methods to establish initial water saturation in core plugs. We used porous plate to obtain uniform initial water distribution along the plug. Brine was drained by a lab oil at pre-defined capillary pressure. This target water saturation was compared with that at the top of the reservoir from saturation logs. The core plug was considered initialized if its water saturation agreed with that from the saturation $\log$ (within \pm 5 saturation unit). Once core plugs were at representative initial water distribution, crude oil was injected into plugs to displace the lab oil at reservoir temperature and a pore pressure of 200 psi. The aging process started; core plugs with crude 
oil were maintained at reservoir temperature and net confining stress for four weeks, as suggested by a previous study [2]. After aging, the crude oil was displaced by the lab oil. Effluent and delta pressure across a core plug were monitored closely to ensure the complete displacement of the post-aging crude. Delta pressure approached stable reading with less noise when the crude oil in the core plug was displaced in the core sample. The clear effluent also confirmed it. Effective permeability to oil at initial water saturation was also measured before and after aging as QC check. At this stage, core plugs prepared at Swi with wettability restored were ready for the SS or centrifuge tests.

\subsection{Supplementary QC tests}

As mentioned above, subsurface conditions like high temperature and high salinity have made core measurements very challenging. Before the actual relative permeability tests, we implemented a few socalled QC tests. The objective was not to measure representative relative permeability data, but to evaluate the test equipment and the test design. As such, crude oil and plugs from the same core were used. QC core plugs followed similar but accelerated preparation procedures. For example, the initial water saturation was obtained by centrifuge desaturation, and the aging duration was shortened to one week. Those QC SS or centrifuge tests were carried out ahead of tests on the actual plugs, and the results were used to optimize test parameters, such as speed selection in centrifuge tests and oil-water injection ratios in SS tests.

\subsection{Relative permeability and capillary pressure tests}

Fourteen core plugs were split into two groups after aging: seven for centrifuge tests and seven for SS tests. Two centrifuge tests were performed on each of the seven plugs sequentially to measure imbibition capillary pressure and secondary drainage capillary pressure, respectively. Eight speeds were used in each multispeed centrifuge test ranged from 400 to $10000 \mathrm{rpm}$ (revolution per minute). Bond number at highest speed was checked to avoid over-desaturation. In the previous program, a Bond number of 10-5 was used, resulting in a very low speed $(<4000 \mathrm{rpm})$ and over-estimated residual saturation. Study $[3,4]$ has shown that the critical Bond number could be orders of magnitude higher than 10-5 for a non-water-wet system. Besides, numerical simulations and QC test results were used to ensure those speeds were reasonably spaced to measure the full capillary pressure curve. During the measurements, we reviewed the automatic recorded raw production data at each speed to assess equilibrium, before moving to the next speed. In the SS tests, water and oil were injected into plugs at nine fractional flow rates: $0,0.01,0.05$, $0.15,0.5,0.85,0.95,0.99$, and 1 . Total injection rate was selected based on the permeability of each individual plug. In each test, once the pressure and saturation were stabilized at $100 \%$ water fractional flow, the injection rate was increased up to five times (ie. bump flood) to reduce any possible capillary end effect. During the test, pressure drop across the plug was closely monitored and multiple X-ray scans were performed at each ratio. The raw data at each ratio was reviewed to assess if a true steady state was achieved before proceeding to the next ratio.

\section{Data analysis}

To obtain capillary pressure and relative permeability data from centrifuge and steady state tests, we started with the analytical calculation followed by numerical simulations. A one-dimensional numerical model was built in MoReS, a Shell in-house simulator [5], for data interpretation. There were 50 grid blocks along the flow/ displacement direction (ie. $\mathrm{z}$ direction). Cross sectional area in the $\mathrm{x}$ and $\mathrm{y}$ plane matched that of a core plug. In a numerical centrifuge test, corresponding centrifugal acceleration was imposed at each speed, and production was simulated. In a steady state test, injection rate and oil-water ratio were imposed. Simulated pressure across a core plug and saturation profiles were monitored. See Maas and Schulte 1997 for more details [6]. As mentioned in the Introduction, SS technique can provide oil-water relative permeability in the intermediate saturation range. The data should be complimented by the residual oil saturation from a centrifuge test to define the full range of relative permeability values. On the other hand, only tail-end of oil relative permeability can be extracted from a centrifuge test. To extract a set of relative permeability and capillary pressure data that simultaneously matches both SS and centrifuge tests, data interpretation was carried out in iterations. Capillary pressure (Pc) was first calculated from centrifuge production data using the Hassler-Brunner method [7]. Oil-water relative permeability (kr) was calculated from Darcy's Law from SS data. The analytical results (Pc and $\mathrm{kr}$ ) were then used as inputs and adjusted to match the actual production data using numerical simulation. Figure 6 shows the delta pressure across the plug and 
saturation profiles in steady state test on sample SS1 matched with numerical simulation. Figures 7 and 8 are the production data from multispeed centrifuge imbibition and secondary drainage tests on sample Cfg1 matched with numerical simulations. One common set of relative permeability and capillary pressure curves had been used to interpret the test results on SS1 and Cfg1 (Figure 9). In the Figure 9 (a), symbols with dashed lines are relative permeability used in simulations, where the residual oil saturation is obtained from centrifuge imbibition Pc measurement. Also shown in Figure 9 are the relative permeability curves derived analytically (open symbols). In this case, when the capillary end effect is small, the analytical and simulation solutions are very similar.

\section{Discussion}

\subsection{Imbibition $P_{c}$ and wettability}

Imbibition Pc curves are plotted in Figure 10. As shown in the figure, the Pc curve is negative with hardly any spontaneous imbibition of water. Average residual oil saturation is around $22 \%$. Characteristics of imbibition Pc curves indicated non-water wet conditions. However, the curve shape and the absolute values did not suggest strongly oil-wet condition either. Figure 11 shows the secondary drainage Pc curves; no oil imbibition was observed. This is a common observation in many sandstone and carbonate samples. Any wettability index calculation from such imbibition and secondary drainage Pc curves may be misleading. It could even lead to a positive wettability index and the erroneous assumption that the reservoir is water-wet which is not the case.

\subsection{Relative permeability}

Oil-water relative permeability curves combining SS and centrifuge results are in Figure 12. Seven sets of relative permeability curves appeared consistent. A range of Corey parameters could be extracted: Sor: $0.19-0.25$, kro(Swi): $0.55-0.65$, krw(Sor): $0.18-0.24$, no: $2.5-$ 2.9 and nw: $2.0-2.6$. Solid lines in the figure describe the average trend by the following Corey parameters: Sor $\sim 0.22, \operatorname{kro}($ Swi $) \sim 0.6, \mathrm{krw}($ Sor $) \sim 0.2$, no $\sim 2.9$, and nw $\sim 2.3$. Figure 13 compares $\mathrm{kro}(\mathrm{Swi})$ and Sor from this work with those from the previous programs. Both parameters from previous programs varied in wide ranges, leading to large uncertainties in reservoir wettability and flow evaluation. Such comparison for other Corey parameters could not be made due to compromised data quality in the previous programs.

\subsection{Reconciliation of imbibition $P_{c}$ and $K_{r}$}

For this set of core samples, measured imbibition Pc indicated that they were non-water-wet. In SS tests, saturation profiles at each ratio appeared relatively flat. Possible interference from the end-pieces could have distorted the profiles near core end-faces. Relatively flat saturation profiles also suggest that the impact of imbibition Pc was minimal. Sample heterogeneity and the viscous pressure drop during steady state tests could have contributed to the observed flat saturation profiles. In steady state tests in this work, pressure drop across the core plug was higher than the imbibition Pc for water saturation up to $\sim 70 \%$. The impact of Pc on the fluid distribution was greatly reduced. CT images of core plugs also showed fine laminations (Figure 3), suggesting varying grain sizes. Thin sections of plug end-trims indicated the alternating coarser-grained and finergrained laminae. Figure 14 is a thin section image of the end-trim from sample SS1. The apparent bi-model distribution of grain sizes derived from the thin section confirmed this alternation. The laminations could have affected the flow during the SS tests. A centrifuge test, in contrast, is a gravity stable displacement and is less affected by heterogeneity. Further investigation is needed to evaluate the potential impact of fine laminations on fluid flow and relative permeability, and the effort is ongoing.

\section{Conclusion}

In this paper, we presented an experimental program to measure relative permeability on core plug samples from a deep-water sandstone reservoir in the Gulf of Mexico. Previous SCAL measurements showed data with compromised quality, due to challenging experimental conditions and lack of data QAQC. In the program execution, we found that:

- Combination of steady state and centrifuge techniques better measures full relative permeability curves.

- Saturation profiles are necessary to evaluate the fluid flow and distribution along the core sample.

- Supplementary tests to assess fluid compatibility and measurement protocol add values to optimize the test design. 
- Numerical simulations include both capillary pressure and relative permeability effects, and provide representative interpretation.

- Continuous and timely monitoring of experimental data is essential to obtain quality data.

We would like to thank Xudong Jing, Keith Love, Menno Hartemink, David Janson, and Marzena Jaminski for insightful discussions.

\section{References}

1. Masalmeh, S.K. and Jing, X.D., "The Importance of Special Core Analysis in Modelling Remaining Oil Saturation in Carbonate Fields", SCA 2008-3, the SCA Annual Symposium, Abu Dhabi, October 2008.

2. Kokkedee, J.A and Boutkan, V.K., "Towards Measurement of Capillary Pressure and Relative Permeability at Representative Wettability", proceedings of the Seventh European IOR Symposium, Moscow, October 1993.

3. Masalmeh, S.K., "Determination of Waterflooding Residual Oil Saturation for Mixed to Oil-Wet
Carbonate Reservoir and its Impact on EOR", SPE165981, SPE Reservoir characterisation and Simulation Conference, Abu Dhabi, September 2013.

4. Humphry, K.J., Suijkerbuijk, B.M.J.M., van der Linde, H.A., Pieterse, S.G. and Masalmeh, S.K., "Impact of Wettability on Residual Oil Saturation and Capillary Desaturation Curves", Petrophysics, 55, 313 (2014)

5. Regtien, J.M.M, Por, G.J.A., van Stiphout, M.T. and van der Vlugt, F.F., "Interactive Reservoir Simulation”, SPE29146, SPE Reservoir Simulation Symposium, San Antonio, USA 1995

6. Maas, J.G. and Schulte, A.M., "Computer Simulation of Special Core analysis (SCAL) Flow Experiments Shared on the Internet", SCA-9719, the SCA Annual Symposium, Canada, 1997.

7. Hassler, G.L and Brunner, E., "Measurement of Capillary Pressures in Small Core Samples", Trans. AIME, 160, 114 (1945)

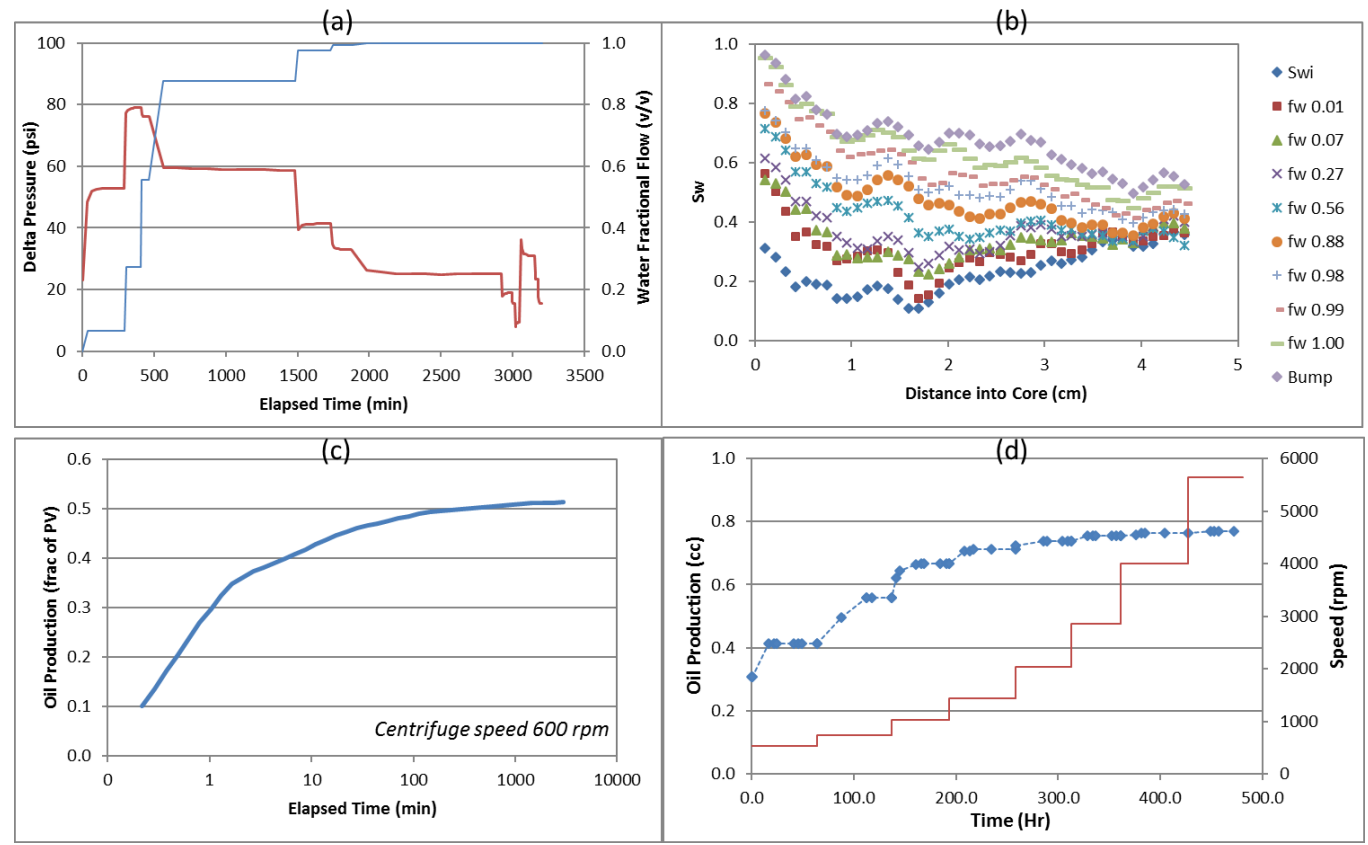

Figure 2. Examples of steady-state (top row) and centrifuge (bottom row) test data previously measured on core plugs from the same area. Several issues were identified from those tests. See text for detailed explanation. 


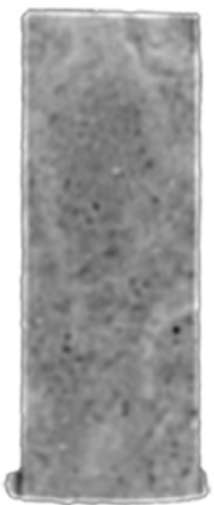

\section{SS1}

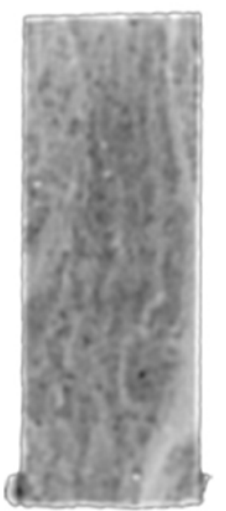

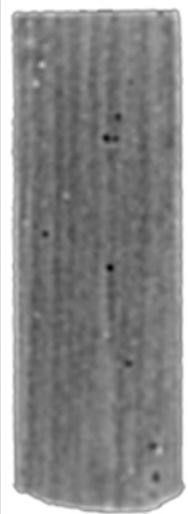

SS4

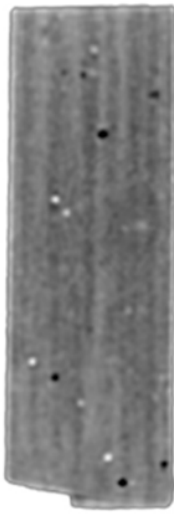

Figure 3. CT images of core plug SS1 and SS4, showing two types of fabrics. Gray scale was adjusted to highlight any fine laminations or density contrast in core plugs.

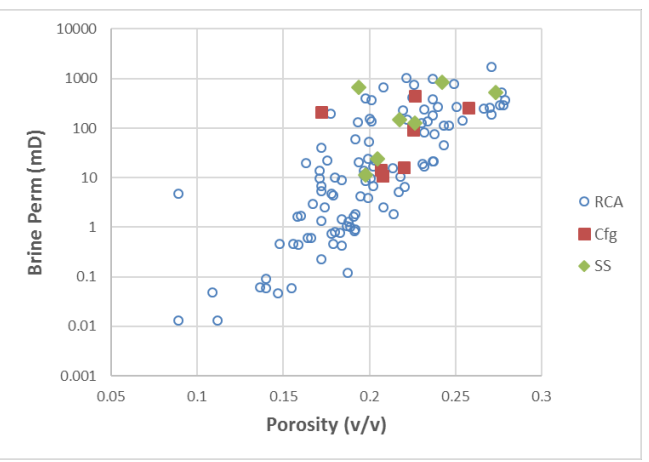

Figure 4. Porosity and permeability of plugs from routine core analysis (RCA) and those for relative permeability tests (Rel Perm).

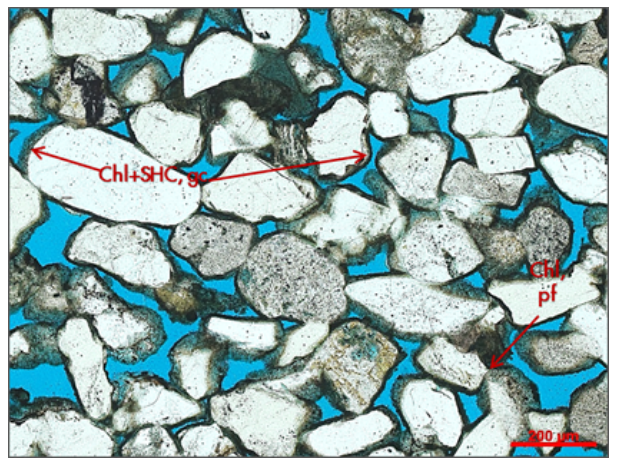

Figure 5. Thin section of the end-trim from core sample Cfg4, where "Chl+SHC, gc" marks the grain-coated chlorite and solid HC.
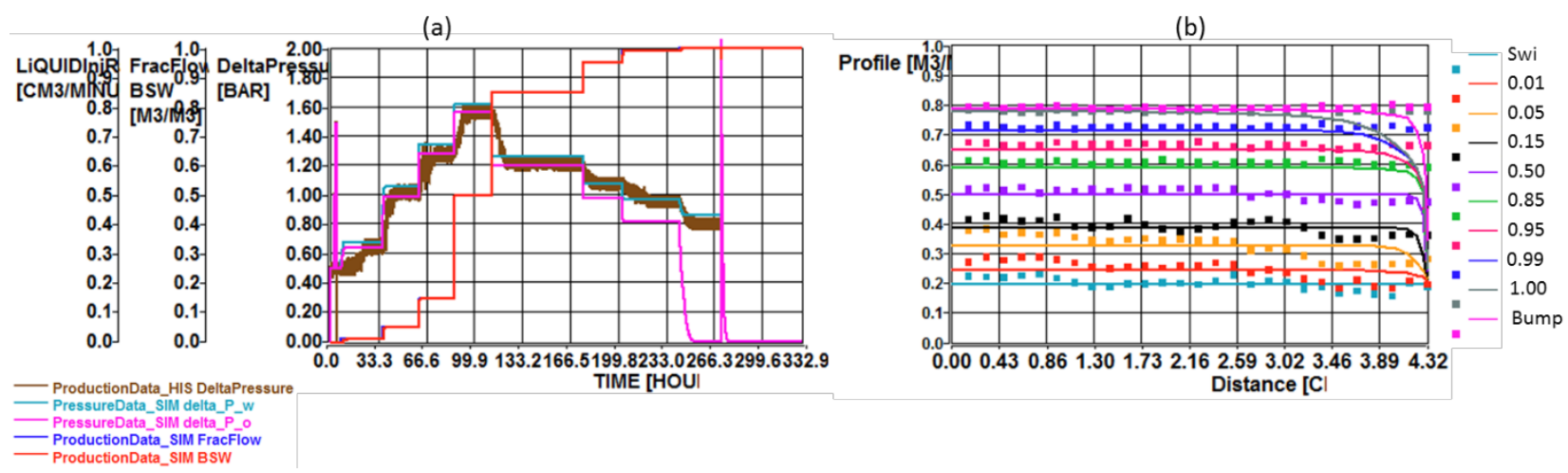

Figure 6. Steady state test results for sample SS1. (a) The measured delta pressure (brown line) was matched by the simulated pressures (light blue and pink lines). (b) The measured water saturations (symbols) were matched by simulations (lines with corresponding colors). 


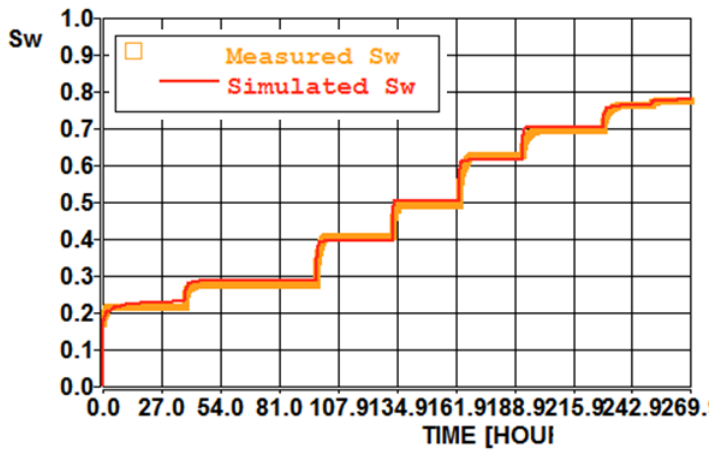

Figure 7. Oil production (in term of water saturation $\mathrm{Sw}$ ) from centrifuge imbibition test on sample Cfg1 (yellow squares) was matched with simulation results (red line).

(a)

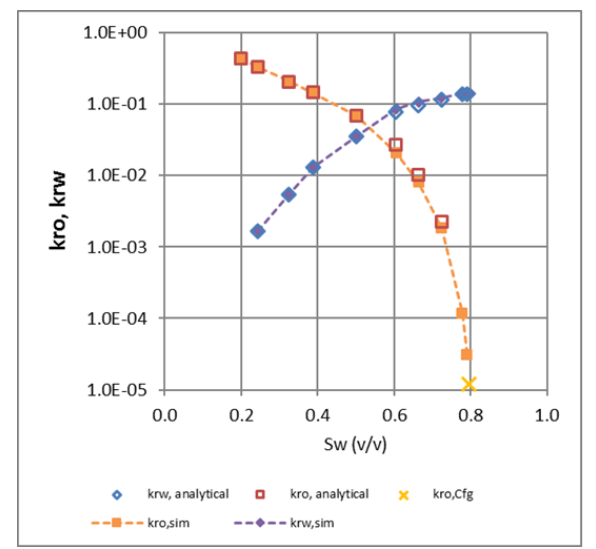

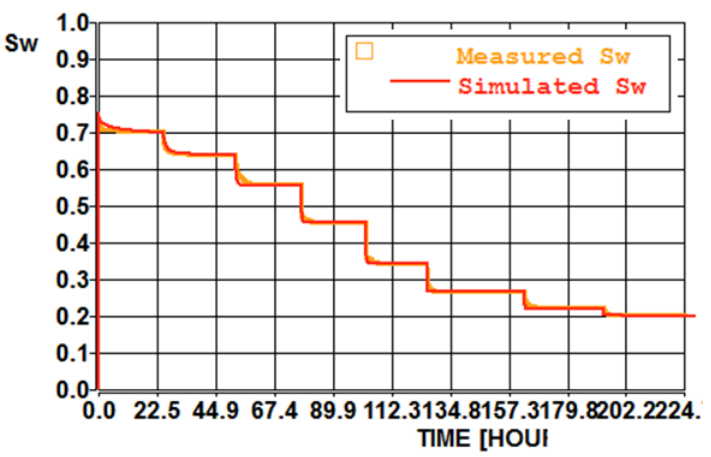

Figure 8. Water production from centrifuge secondary drainage test on sample Cfg1 (yellow squares) was matched with simulation results (red line).

Figure 9. Imbibition oil-water relative permeability (a) and capillary pressure (b) curves derived from steady state test on SS1 and centrifuge test on Cfg1. See text for explanation.

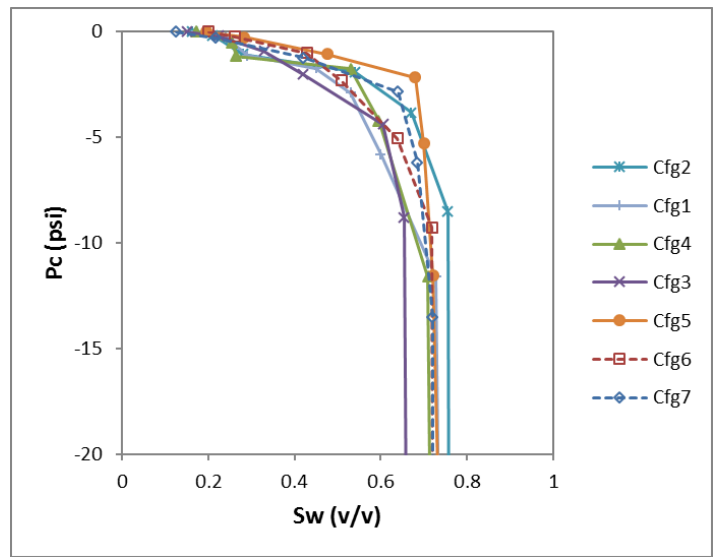

Figure 10. Imbibition capillary pressure curves interpreted from multispeed centrifuge tests.

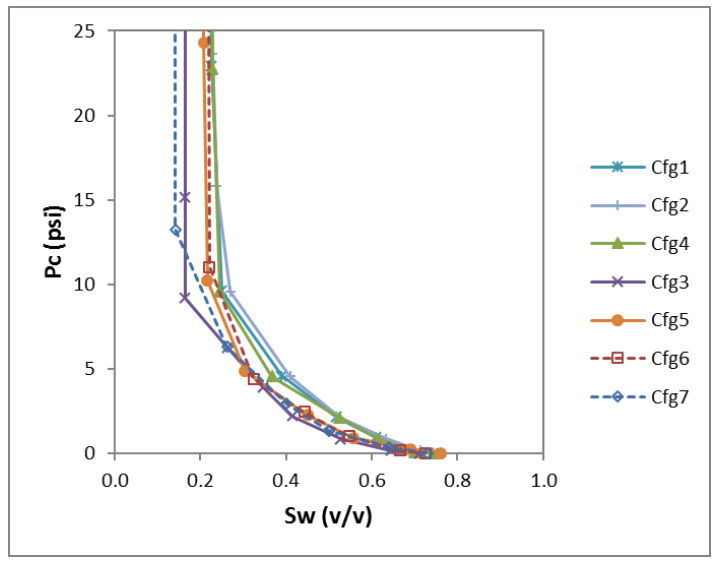

Figure 11. Secondary drainage capillary pressure curves interpreted from multispeed centrifuge tests. 

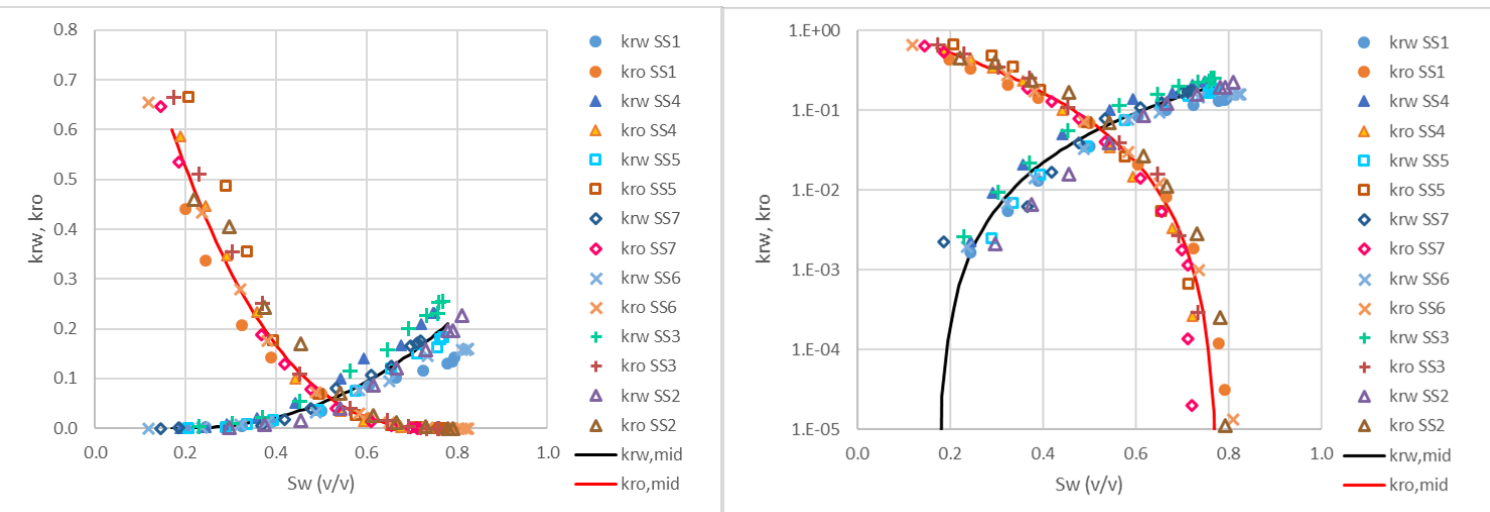

Figure 12. Relative permeability curves combining both steady state and centrifuge test results in linear scale (left) and semi-log scale (right).

(a)

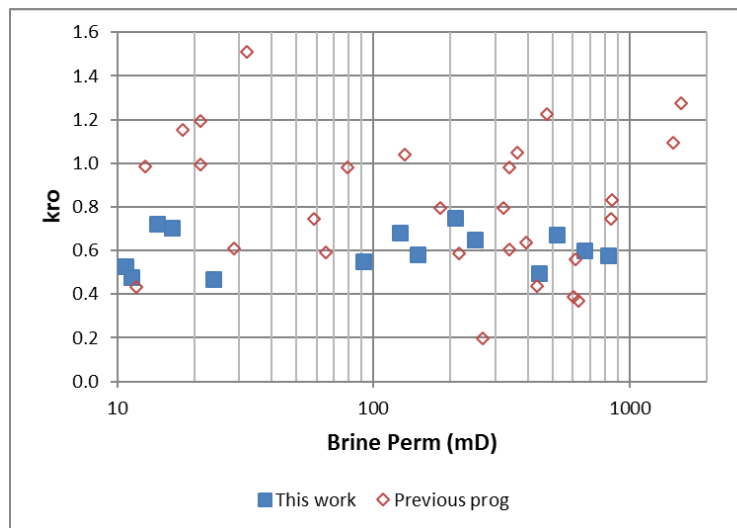

(b)

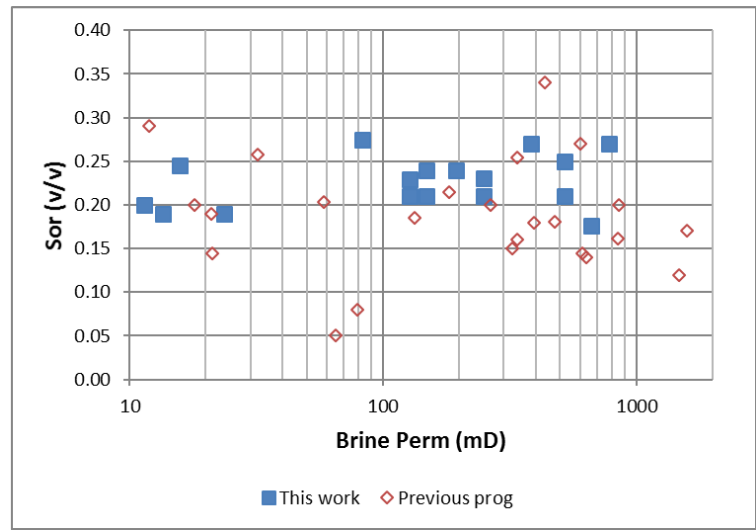

Figure 13. Comparison of kro(Swi) and Sor from this work with those from the previous programs.
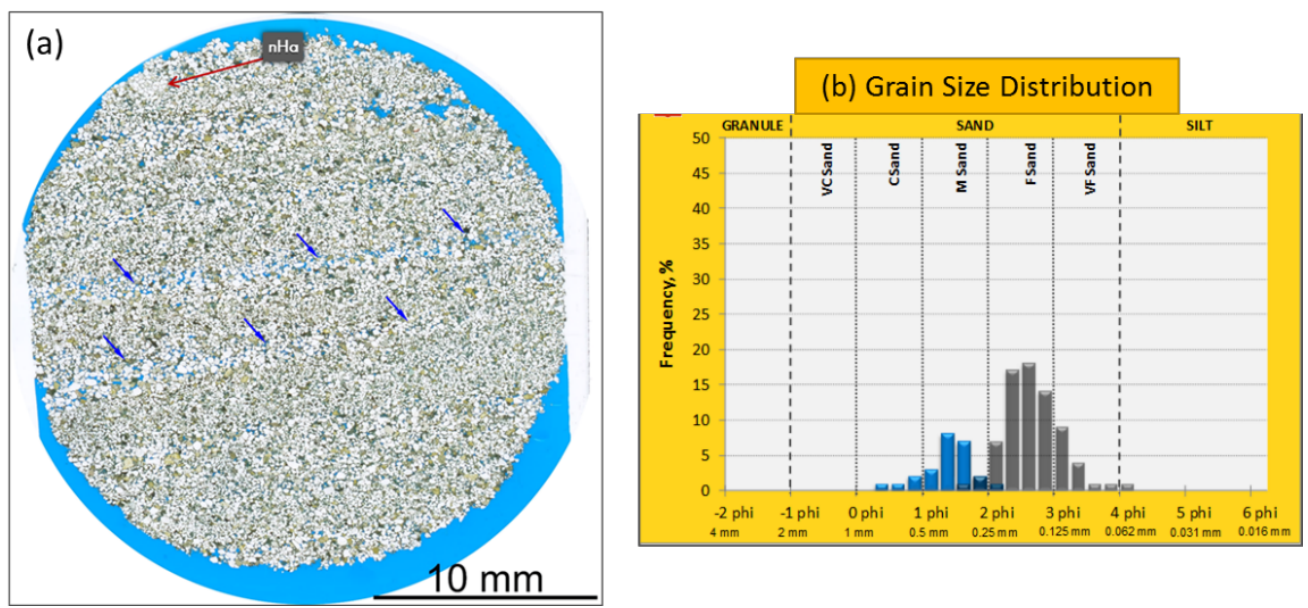

Figure 14. (a) Thin section image of the end-trim from sample SS1, where blue arrows mark the coarser-grained laminae. (b) Grain sizes derived from thin section suggests bi-modal distribution. 